\title{
Glucocorticoid treatment inhibits intracerebral hemorrhage-induced inflammation by targeting the microRNA-155/SOCS-1 signaling pathway
}

\author{
HONG-FEI XU ${ }^{1 *}$, XIAO-YUN FANG ${ }^{2 *}$, SHAO-HUA ZHU ${ }^{1}, X^{*}$ XE-HUA XU ${ }^{1}$, \\ ZHI-XIANG ZHANG ${ }^{1}$, ZU-FENG WANG ${ }^{1}$, ZI-QIN ZHAO ${ }^{3}$, YU-JIE DING ${ }^{4}$ and LU-YANG TAO ${ }^{1}$
}

${ }^{1}$ Department of Forensic Medicine, Soochow University, Suzhou, Jiangsu 215123; ${ }^{2}$ Jiangsu Patent Examination Assistance
Center Under State Intellectual Property Office of The People's Republic of China, Suzhou, Jiangsu 215163;
${ }^{3}$ Department of Forensic Medicine, Shanghai Medical College, Fudan University, Shanghai 200032;
${ }^{4}$ Department of Dermatology, The Second Affiliated Hospital of Soochow University, Suzhou, Jiangsu 215123, P.R. China

Received August 31, 2015; Accepted June 8, 2016

DOI: $10.3892 / \mathrm{mmr} .2016 .5716$

\begin{abstract}
Intracerebral hemorrhage (ICH) results in inflammation, and glucocorticoids have been proven to be effective inhibitors of ICH-induced inflammation. However, the precise underlying mechanisms of ICH-induced inflammation and glucocorticoid function remain largely undefined. Using a mouse ICH model, the present study demonstrated that the short non-coding RNA molecule microRNA-155 (miR-155) is involved in the inflammatory process initiated by ICH in mice. Increased mRNA expression levels of miR-155, as well as the pro-inflammatory cytokines interferon- $\beta$ (IFN- $\beta$ ), tumor necrosis factor- $\alpha$ (TNF- $\alpha$ ) and interleukin-6 (IL-6), were observed in vivo following ICH. By contrast, the expression level of suppressor of cytokine signaling 1 (SOCS-1) protein was reduced in the ICH group compared with control mice. Similar results were observed in vitro using astrocytes, the primary effector cells in ICH. Compared with wild type astrocytes, astrocytes overexpressing miR-155 exhibited significant inhibition of SOCS-1 protein expression levels. These results suggest that miR-155 contributes to the development of ICH-induced inflammation in mice by downregulating SOCS-1 protein expression levels and promoting pro-inflammatory
\end{abstract}

Correspondence to: Dr Yu-Jie Ding, Department of Dermatology, The Second Affiliated Hospital of Soochow University, 1055 SanXiang Road, Suzhou, Jiangsu 215123, P.R. China

E-mail: 45757669@qq.com

Professor Lu-Yang Tao, Department of Forensic Medicine, Soochow University, Suzhou Dushuhu High Educational Town, 138 RenAi Road, Suzhou, Jiangsu 215123, P.R. China

E-mail: 41845875@qq.com

*Contributed equally

Key words: microRNA-155, intracerebral hemorrhage, suppressor of cytokine signaling 1, signaling pathway, glucocorticoid cytokine (IFN- $\beta$, TNF- $\alpha$ and IL-6) production. Expression levels of miR-155 and pro-inflammatory cytokines in the $\mathrm{ICH}$ group were significantly decreased following dexamethasone administration. This suggests that glucocorticoids attenuate ICH-induced inflammation by targeting the miR-155/SOCS-1 signaling pathway in mice. In conclusion, the results of the present study demonstrated that the miR-155/SOCS-1 signaling pathway is required for $\mathrm{ICH}$-induced inflammation, and glucocorticoids inhibit this process by targeting the miR-155/SOCS-1 signaling pathway.

\section{Introduction}

Intracerebral hemorrhage (ICH) is a common and severe subtype of stroke associated with high rates of morbidity and mortality (1). Resulting from ruptured blood vessel(s) in the brain, ICH is defined as bleeding into the brain parenchyma (2). A series of pathophysiological processes occurs following an acute ICH outbreak, including apoptosis and necrosis, breakdown of the blood-brain barrier, edema formation, inflammation and extracellular matrix remodeling (1). These complications may result in severe neuronal injury. Thus, it is important to understand the underlying mechanisms of ICH in order to control the subsequent complications. Despite this, relatively few studies have focused on ICH and its subsequent effects compared with studies on ischemic stroke (3-5).

Characterized by elevated expression levels of pro-inflammatory cytokines, including interferon- $\beta$ (IFN- $\beta$ ), tumor necrosis factor- $\alpha$ (TNF- $\alpha$ ) and interleukin 6 (IL-6), inflammation induced by $\mathrm{ICH}$ is hypothesized to contribute to neurodegeneration $(6,7)$. Glucocorticoids (GCs) exert anti-inflammatory effects, and are therefore considered to be effective therapeutic agents in acute and chronic inflammation, including severe shock, asthma, inflammatory bowel disease, rheumatoid arthritis and multiple sclerosis (8-10). In addition, studies investigating their efficacy in the treatment of ICH-induced inflammation have revealed positive results (11-13). However, the underlying mechanisms through which GCs exert their anti-inflammatory and immunosuppressive functions in ICH remain to be elucidated (14). 
MicroRNAs (miRs) are endogenous 20-23 nucleotide small noncoding RNAs that are expressed in various tissues and are responsible for regulating the expression of various genes. miRs affect numerous biological processes, including development, autoimmune diseases, inflammation and proliferation (15-19). miRs function by shortening mRNA half-life or inhibiting translation via binding the 3 'untranslated region of target genes (20). miR-155 is encoded by the B-cell integration (BIC) gene located on chromosome $21(20,21)$. Previous studies have suggested that miR-155, similar to other miRs, is closely associated with the development of inflammatory pathogenesis (22). Furthermore, Liu et al (23) revealed the involvement of miR-155 in rat ICH.

Previous studies have focused on the miR-155-mediated signaling pathway, in order to elucidate its precise underlying molecular mechanisms in various biological processes $(20,21)$. Identified targets of miR-155 to date include Fas-associated death domain protein, Src homology 2-containing inositol 5-phosphatase 1 and the suppressor of cytokine signaling-1 (SOCS-1) (20,24-26). In microglia-mediated immune reactions, miR-155 promoted inflammation via the downregulation of SOCS-1 protein (6). However, the underlying mechanisms by which miR-155 functions in ICH-induced inflammatory pathogenesis remain to be elucidated.

In the present study, a mouse ICH model was established according to our previous paper $(27,28)$ and miR-155 was overexpressed in astrocytes to investigate the role of miR-155 in ICH-induced inflammation, as well as the underlying mechanism of the anti-inflammatory effects of GCs.

\section{Materials and methods}

Animals and ICH-induction. All experimental procedures were in compliance with the National Institutes for Health Guide for the Care and Use of Laboratory Animals (29) and approved by the Institutional Animal Care and Use Committee at Soochow University (Suzhou, China). Animals, including both mice and rats, were housed under pathogen-free conditions with 10:14 h light: Dark cycle, at room temperature and $75 \%$ humidity. Animals had free access to sterile water and chow. Protocols for ICH-induction were conducted as described previously $(27,28)$. Briefly, 30 adult male ICR mice weighing 25-30 g and 6 neonatal Sprague-Dawley rats were obtained from Shanghai Laboratory Animal Center (Shanghai, China). ICH was induced by collagenase injection, as previously described (27). Mice were anesthetized with $4 \%$ chloral hydrate $(0.4 \mathrm{mg} / \mathrm{g})$ purchased from Sigma-Aldrich (St. Louis, MO, USA) and placed in a stereotactic apparatus. A cranial burr hole (1-mm in diameter) was drilled, through which type IV collagenase purchased from Sigma-Aldrich was injected ( 0.075 units in $500 \mathrm{nl}$ of saline) into the left striatum ( $1 \mathrm{~mm}$ anterior and $2 \mathrm{~mm}$ lateral of the bregma, $3.5 \mathrm{~mm}$ in depth). Collagenase was delivered over $5 \mathrm{~min}$. The needle was kept in place for an additional 5 min to prevent any reflux. The control (sham) mice received an equivalent volume of sterile saline injected in an identical manner. Body temperature was maintained at $37 \pm 0.5^{\circ} \mathrm{C}$ using a heat lamp throughout surgery and recovery.

Experimental groups and drug administration. Mice were randomly assigned to three groups: control (Ctrl), Sham surgery (sham) and ICH, with 6 mice per group. To investigate the effect of GCs, dexamethasone $(30 \mathrm{mg} / \mathrm{kg})$ was administered via i.p. injection twice a day for 3 consecutive days, beginning on the day of surgery. Animals were sacrificed one day after the last dose, a total of three days following surgery. Animals were sacrificed by an intraperitoneal injection of pentobarbital (40 mg/kg, Sigma-Aldrich) and brain tissues were collected for analysis. Dexamethasone was purchased from Sigma-Aldrich and administered according to previously published proto$\operatorname{cols}(27,30)$.

RNA isolation. Total RNA was extracted from brain tissue samples or cultured astrocytes using TRIzol ${ }^{\circledR}$ Reagent (Invitrogen; Thermo Fisher Scientific, Inc., Waltham, MA, USA), according to the manufacturer's instructions. Any remaining DNA was removed with the DNA-free kit (Ambion; Thermo Fisher Scientific, Inc.) and RNA was purified with an RNeasy kit (Qiagen, Inc., Valencia, CA, USA), according to the manufacturer's instructions.

Detection of miR-155 by reverse transcription-quantitative polymerase chain reaction $(R T-q P C R)$. For quantification of miR-155 levels, RT-qPCR was performed on total RNA. miR-155 expression levels were determined using a Hairpin-it ${ }^{\mathrm{TM}}$ miRs qPCR kit (Shanghai GenePharma Co., Ltd., Shanghai, China). In brief, the assay has two steps: Stem-loop RT reaction and qPCR detection. Stem-loop RT primers bind to the $3^{\prime}$ end of miR molecules and are transcribed with reverse transcriptase. Briefly, the total RNA was reverse transcribed using the M-MLV reverse transcriptase system (Promega Corporation, Madison, WI, USA). The reaction was performed at $42^{\circ} \mathrm{C}$ for $1 \mathrm{~h}$ and terminated by deactivation of the enzyme at $70^{\circ} \mathrm{C}$ for $10 \mathrm{~min}$. The RT product was quantified by qPCR, using miR-specific forward and reverse primers as follows: Forward, 5'-GTGCTGCAAACCAGGAAGG-3' and reverse, 5'-CTGGTT GAA TCA TTGAAGATGG-3', and a carboxyfluorescein dye-labeled reporter probe. Thermal cycling conditions were as follows: Pre-denaturation at $95^{\circ} \mathrm{C}$ for $5 \mathrm{~min}$, denaturation at $95^{\circ} \mathrm{C}$ for $10 \mathrm{sec}$, annealing at $58^{\circ} \mathrm{C}$ for $15 \mathrm{sec}$, and extension at $72^{\circ} \mathrm{C}$ for $20 \mathrm{sec}$. Gene expression levels were standardized to a housekeeping gene (U6) and expressed as a fold of control. To normalize RNA content, the U6 small nuclear RNA served as the internal control. The relative miR-155 expression levels of each group were calculated using the $\Delta \Delta \mathrm{Cq}$ method (31).

Cytokine detection was performed by RT-qPCR using an ABI 7300 RT-PCR instrument (Applied Biosystems; Thermo Fisher Scientific, Inc.) using a SYBR Green-based RT-PCR kit [SYBR ${ }^{\circledR}$ Premix Ex Taq ${ }^{\mathrm{TM}}$ (Tli RNaseH Plus); Takara Bio, Inc., Otsu, Japan]. The primers used were as follows: IFN- $\beta$ (32) forward, 5' AAGAGTTACACTGCCTTTGCCATC3' and reverse, $5^{\prime}$ 'CACTGTCTGCTGGTGGAGTTCATC 3 '; TNF- $\alpha$ forward, 5'TGAACTTCGGGGTGA TTGGTC3' and reverse, 5'GCCTTGTCCCTTGAAGAGAAC3'; IL-6 forward, 5'-TAC TTCACAAGTCCGGAG-3' and reverse, 5'-TCCAGAAGACCA GAGCAG-3'; $\beta$-actin forward, 5'-ACATCTGCTGGAAGG TCCAC-3' and reverse, 5'-GGTACCACCATGTACCCAGG-3'. All reactions were performed three times for each group.

Western blot analysis of SOCS-1 protein expression levels. Protein extract prepared from tissue samples or cultured astro- 
cytes were examined by western blot analysis. Protein was extracted using the ProteoExtract ${ }^{\circledR}$ Transmembrane Protein Extraction kit (Merck Millipore, Darmstadt, Germany) according to the manufacturer's protocol, and determination of protein concentration was performed using a bicinchoninic acid protein assay kit (Pierce; Thermo Fisher Scientific, Inc.) according to the manufacturer's protocol. Equal amounts of protein $(15 \mu \mathrm{g})$ were subjected to $10 \%$ SDS-PAGE (running at $60 \mathrm{~V}$ for $1 \mathrm{~h}$ through the stacking gel, and at $120 \mathrm{~V}$ for $1 \mathrm{~h}$ through the resolving gel). Western blot analysis was performed using a mouse antibody against SOCS1 (1:1,000 dilution, catalog no. 04-002, EMD Millipore, Billerica, MA, USA) (27). Briefly, proteins were blotted onto a polyvinylidene difluoride membrane (Roche Applied Science, Penzburg, Germany) for $90 \mathrm{~min}$ at $350 \mathrm{~mA}$. Then the membranes were blocked with $0.1 \%$ Tris-buffered saline (TBS)-Tween buffer containing $5 \%$ skim milk either overnight at $4^{\circ} \mathrm{C}$ or for $2 \mathrm{~h}$ at room temperature. The membrane was subsequently incubated with anti-SOCS1 antibody overnight at $4^{\circ} \mathrm{C}$. Following the incubation, the membrane was washed with $0.1 \%$ TBS-Tween buffer and probed with goat anti-mouse HRP-conjugated IgG secondary antibody (1:2,000 dilution; catalog no. sc-2005; Santa Cruz Biotechnology, Inc., Dallas, TX USA) for $1 \mathrm{~h}$ at room temperature. Rabbit antibody against GADPH (1:5,000 dilution; catalog no. KC-5G4; Kangcheng, Inc., Shanghai, China) was used as the loading control. Protein bands were visualized using enhanced chemiluminescence (Vazyme Biotech Co., Ltd., Nanjing, China) (27,33). SOCS-1 expression levels were quantified by densitometry in arbitrary units using ImageJ 1.44p software (National Institutes of Health, Bethesda, MD, USA), and normalized to GAPDH.

Astrocyte isolation and culture. Astrocytes were isolated from mixed glial cultures as described previously (34). Briefly, brains harvested from 1- to 3-day-old Sprague Dawley rat pups were minced, filtered and cultured in Dulbecco's modified Eagle's medium (DMEM)/F-12 (Invitrogen; Thermo Fisher Scientific, Inc.). At $100 \%$ confluence (1 week post-plating), the mixed glial cultures were shaken at $200 \mathrm{rpm}$ overnight at $37^{\circ} \mathrm{C}$ on a rotary shaker to separate the microglia and oligodendrocytes from the adherent astrocytes. The media, microglia and oligodendrocytes were removed; astrocytes were trypsinized and subcultured in DMEM/F-12 supplemented with $10 \%$ fetal bovine serum, $100 \mathrm{IU} / \mathrm{ml}$ penicillin, $100 \mathrm{mg} / \mathrm{ml}$ streptomycin, $100 \mathrm{mg} / \mathrm{ml} \mathrm{L}$-glutamine and $15 \mathrm{mM}$ 4-(2-hydroxyethyl)-1-piperazineethanesulphonic aciction id (all Sigma-Aldrich). Astrocytes were maintained at $37^{\circ} \mathrm{C}$ in $5 \% \mathrm{CO}_{2}$ and $95 \%$ air for 3 days.

Transfection of miRs. miR-155 and its mutant constructs were synthesized by Integrated DNA Technologies, Inc., Coralville, IA, USA. The sequences of anti-miR-155 antisense inhibitor oligonucleotides (AMOs) used in the present study are exact antisense copies of mature miR sequences, and the nucleotides in the AMOs contain 2'-O-methyl modifications at every base and a 3'C3 amino linker (31). Oligo transfection was performed according to an established protocol (35). Briefly, wild type (WT) cells were transfected using PolyFect transfection reagent (Qiagen, Inc.) for $6 \mathrm{~h}$. Transfection complexes were prepared according to the manufacturer's instructions, and
2'OMe-miR-155 or control oligo 2'OMe-enhanced green fluorescent protein was added directly to the complexes to a final oligonucleotide concentration of 30,50 or $100 \mathrm{nmol} / \mathrm{l}$. The transfection efficiency was low at 30 or $50 \mathrm{nmol} / \mathrm{l}$. However, when transfected at $100 \mathrm{nmol} / \mathrm{l}$, transfection efficiency reached $\geq 80 \%$. Therefore, only cells receiving $100 \mathrm{nmol} / 1$ were analyzed. The transfection medium was replaced $6 \mathrm{~h}$ subsequent to transfection with regular culture medium, and cells were cultured for 3 days prior to analysis.

Statistical analysis. Data are presented as the mean \pm standard error. Comparisons were made between groups using the Student's $t$-test using SigmaPlot version 10 software (Systat Software, Inc., San Jose, CA, USA). P $<0.05$ was considered to indicate a statistically significant difference.

\section{Results}

miR-155 is involved in ICH pathogenesis in mice in vivo. To evaluate the role of miR-155 in ICH-mediated inflammation, a mouse model of ICH was used, and the expression of miR-155 was determined by RT-qPCR three days subsequent to $\mathrm{ICH}$ treatment. As presented in Fig. 1A, the expression level of miR-155 in the ICH group was significantly increased compared with the control $(\mathrm{P}=0.030)$ and sham groups $(\mathrm{P}=0.038)$. Furthermore, to identify the signaling pathway mediated by miR-155 in the ICH model, western blotting was performed to examine the SOCS-1 protein expression levels in the three groups. In contrast to miR-155, the expression level of SOCS-1 protein was significantly decreased in the ICH group compared with the control mice ( $\mathrm{P}=0.021$; Fig. 1B). In addition, significantly increased mRNA expression levels of the pro-inflammatory cytokines, IFN- $\beta$, IL- 6 and TNF- $\alpha$ were observed following ICH treatment compared with control groups $(\mathrm{P}=0.005,0.002$ and $\mathrm{P}<0.001$, respectively; Fig. $1 \mathrm{C})$. These data indicate that miR-155 and SOCS-1 are involved in the inflammatory pathogenesis induced by ICH. Furthermore, miR-155 and SOCS-1 are antagonistic in this process.

Dexamethasone inhibits ICH-induced inflammation by targeting miR-155 and SOCS-1. To elucidate the underlying functional mechanism of GCs in ICH-induced inflammation, $30 \mathrm{mg} / \mathrm{kg}$ dexamethasone was administered to mice following ICH induction. A total of three days subsequent to administration, miR-155 expression was determined by RT-qPCR. As presented in Fig. 1A, the level of miR-155 expression in mice treated with dexamethasone (ICH+DEX group) was significantly reduced compared with mice that did not receive dexamethasone (ICH group; $\mathrm{P}=0.042$ ). In addition, ICH-mediated inhibition of the protein expression levels of SOCS-1 was reversed following dexamethasone administration $(\mathrm{P}=0.022$; Fig. 1B). Furthermore, the mRNA expression levels of the cytokines IFN- $\beta$, IL- 6 and TNF- $\alpha$ were markedly decreased in the $\mathrm{ICH}+\mathrm{DEX}$ group compared with the $\mathrm{ICH}$ group ( $\mathrm{P}=0.037,0.024$ and 0.018 , respectively; Fig. $1 \mathrm{C})$.

MiR-155 inhibits the expression of SOCS-1 protein in astrocytes in vitro. As astrocytes are the primary effector cells in the inflammatory pathogenesis triggered by $\mathrm{ICH}$, experiments were performed in vitro using astrocytes to attempt to 
A

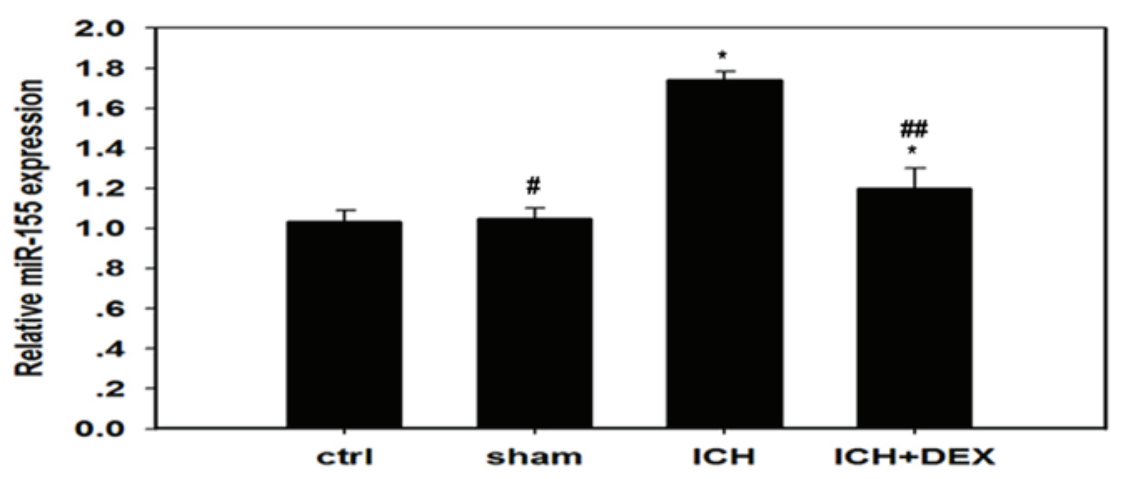

B
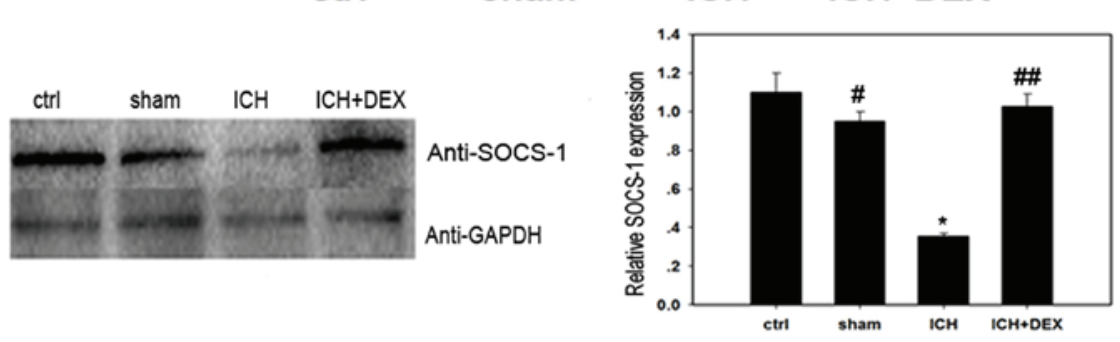

C
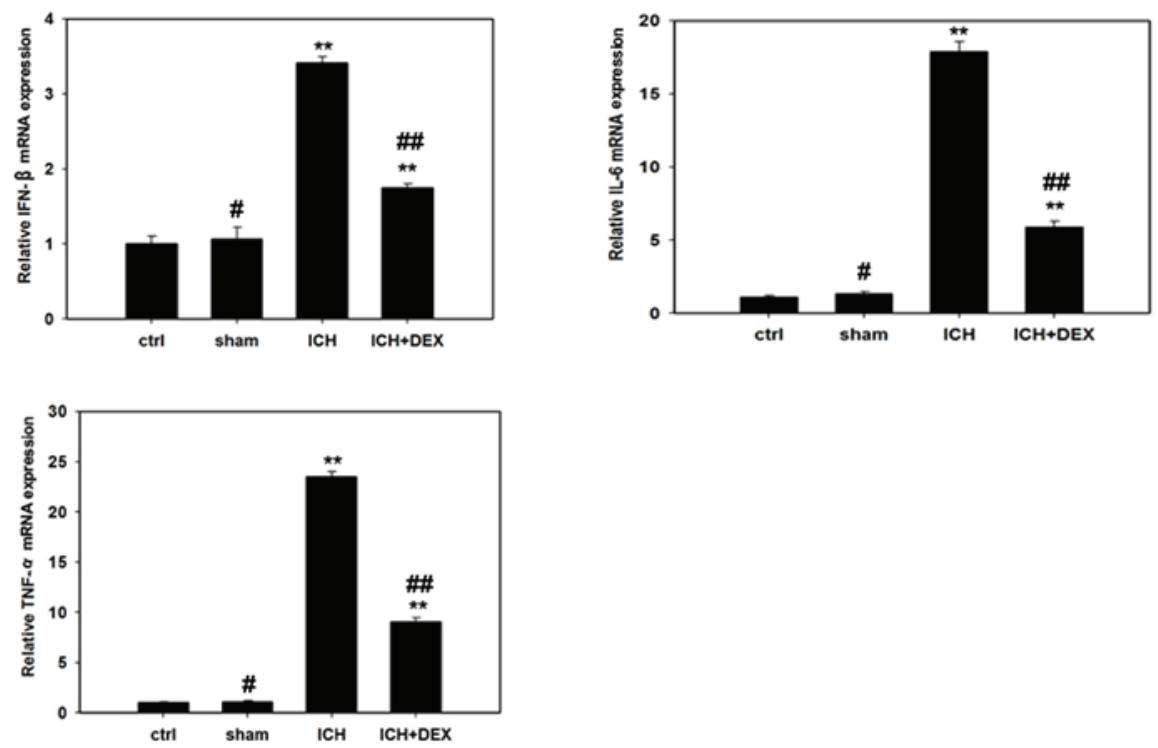

Figure 1. miR-155/SOCS-1 are involved in ICH-induced inflammation and dexamethasone reduces inflammation by inhibiting miR-155 expression in vivo. The expression levels of (A) miR-155, (B) SOCS-1 and (C) pro-inflammatory cytokines were determined under various treatment conditions. Mice were divided randomly into four groups ( $\mathrm{n}=36$ per group): $\mathrm{Ctrl}$ group, without any treatment; ICH group, injected with collagenase for ICH induction: Sham group, injected with the equivalent volume of saline; and ICH+DEX group, treated with dexamethasone following ICH induction. Reverse transcription-quantitative polymerase chain reaction was performed to determine miR-155, IFN- $\beta$, IL- 6 and TNF- $\alpha$ mRNA expression levels, while western blotting determined SOCS- 1 protein expression levels. Representative data is presented, as the mean \pm standard error, from one of at least three experiments. ${ }^{*} \mathrm{P}<0.05 \mathrm{vs}$. ctrl; ${ }^{* *} \mathrm{P}<0.01 \mathrm{vs}$. ctrl (for ICH group) or $\mathrm{P}<0.01$ vs. ICH group (for ICH+DEX group); ${ }^{\#} \mathrm{P}>0.05$ vs. ctrl; and ${ }^{\# \#} \mathrm{P}<0.05$ compared with ICH group, as determined by Student's $t$-test using SigmaPlot version 10.0 software. miR, microRNA; SOCS-1, suppressor of cytokine signaling-1; ICH, intracerebral hemorrhage; ctrl, control; DEX, dexamethasone; IFN- $\beta$, interferon- $\beta$; IL- 6 , interleukin-6; TNF- $\alpha$, tumor necrosis factor- $\alpha$.

clarify the association between miR-155 and SOCS-1 in the ICH model. WT astrocytes were transfected with miR-155 oligonucleotides to overexpress miR-155 (miR-155 group) or with mis-miR-155 oligonucleotides represented as scramble $\mathrm{miR}$ as a control (mis-miR-155 group). Transfection efficiency was $>80 \%$ and no clear morphology impairment was observed following transfection (Fig. 2A). The three populations of astrocytes were collected and assayed for miR-155 expression by RT-qPCR (Fig. 2B), and for SOCS-1 expression by western blotting (Fig. 2C). Overexpression of miR-155 led to a significant inhibition in SOCS-1 protein expression levels compared with the control (WT group; $\mathrm{P}=0.027$ ). This indicates that SOCS-1 is a target of miR-155 in astrocytes, and that miR-155 may promote ICH-triggered inflammatory pathogenesis by downregulating SOCS-1 expression.

To confirm the association between miR-155 and SOCS-1, anti-miR-155 antisense inhibitor oligonucleotides (AMO-155) were synthesized to antagonize the expression of miR-155, and mis-AMO-155 oligonucleotides represented as scramble miR served as a control. AMO-155 and miR-155 oligonucleotides were transfected simultaneously into certain astrocytes (AMO-155+miR-155 group) to cancel out the miR-155 expres- 
A

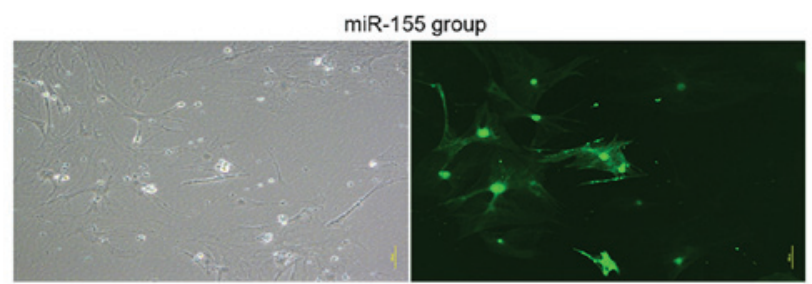

mis-miR-155 group

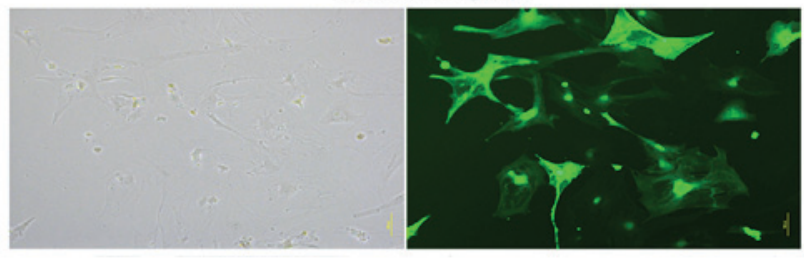

B

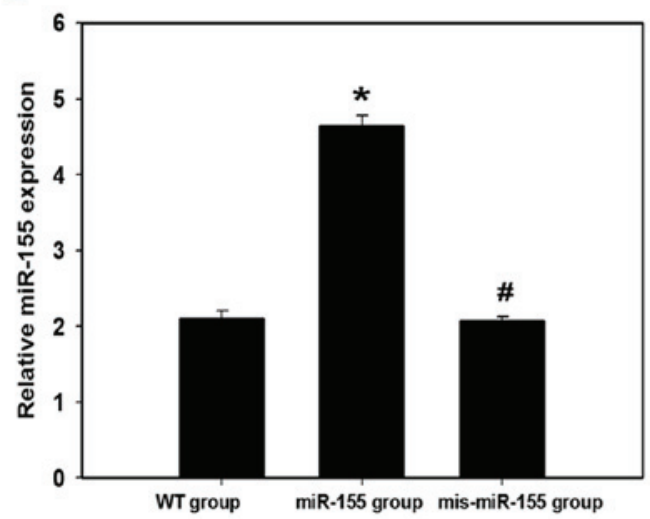

C
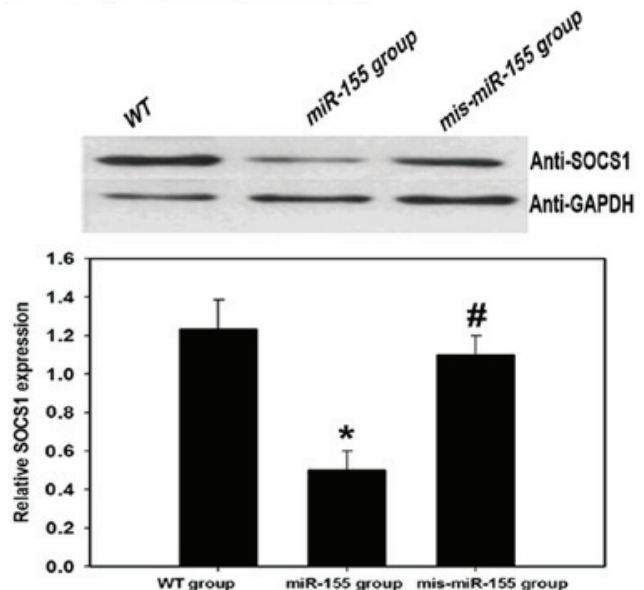

Figure 2. Overexpression of miR-155 results in inhibition of SOCS-1 expression in astrocytes. (A) To obtain miR-155 overexpressing astrocytes, WT astrocytes were cultured and transfected with $100 \mathrm{nmol} / 1 \mathrm{miR}-155$ oligonucleotides for overexpression of miR-155 (miR-155 group) or with $100 \mathrm{nmol} / 1 \mathrm{mis}-\mathrm{miR}-155$ oligonucleotides as a negative control (mis-miR-155 group) for $72 \mathrm{~h}$. Green fluorescence represents a reporter gene. Magnification, x200; scale bar=20 $\mu \mathrm{m}$. Representative results of at least three experiments are presented. (B) Total RNA was extracted from astrocytes and the level of miR-155 expression detected by reverse transcription-quantitative polymerase chain reaction. Values were normalized to U6 expression and presented as the mean \pm SE. Representative results from one of at least three experiments are presented. (C) Western blotting was performed on whole cell lysates to determine SOCS-1 protein expression. SOCS-1 expression levels were quantified by densitometry in arbitrary units using ImageJ software, and normalized to GAPDH. Data are expressed as the mean $\pm \mathrm{SE}$, and representative results are presented from at least three experiments. ${ }^{*} \mathrm{P}<0.05$; and ${ }^{\text {" }} \mathrm{P}>0.05$ compared with WT group, as determined by Student's $t$-test using SigmaPlot version 10.0 software. miR, microRNA; SOCS-1, suppressor of cytokine signaling-1; WT, wild type; SE, standard error.

A

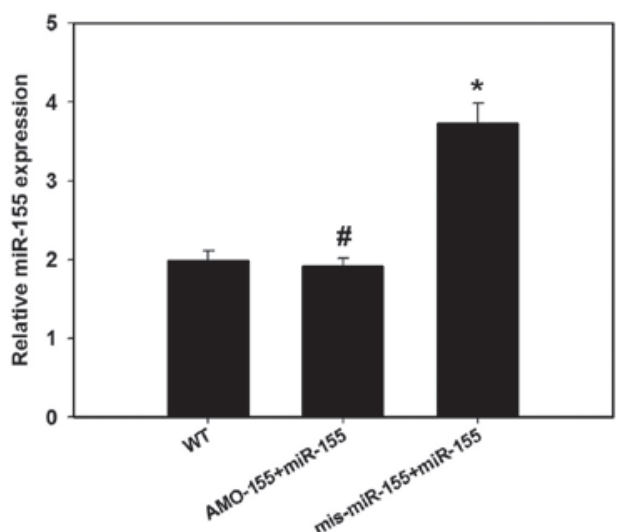

B

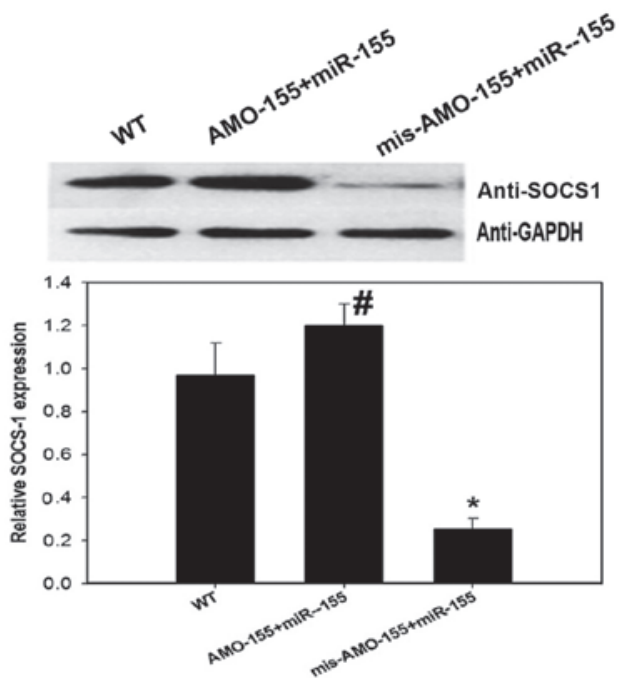

Figure 3. AMO-155 restores SOCS-1 expression in miR-155 overexpressing astrocytes. AMO-155 and mis-AMO-155 oligonucleotides were transfected into astrocytes together with miR-155 oligonucleotides. (A) miR-155 expression levels were determined by reverse transcription-quantitative polymerase chain reaction. (B) SOCS-1 protein expression levels were determined by western blotting. Data are expressed as the mean \pm standard error, and representative results from at least three experiments are presented. "P $<0.05$; and ${ }^{\#} \mathrm{P}>0.05$ compared with WT group as determined by Student's $t$-test using SigmaPlot version 10.0 software. miR, microRNA; SOCS-1, suppressor of cytokine signaling-1; AMO, antisense inhibitor oligonucleotides; WT, wild type. 
sion, while a separate group were transfected withmis-AMO-155 together with miR-155 oligonucleotides to examine the overexpression of miR-155 (mis-AMO-155+miR-155 group). As presented in Fig. 3A, expression levels of miR-155 were increased in the mis-AMO-155+miR-155 group $(\mathrm{P}=0.030)$ although not in the AMO-155+miR-155 group $(\mathrm{P}=0.724)$, compared to WT cells. As presented in Fig. 3B, expression levels of SOCS-1 protein were significantly inhibited in the mis-AMO-155+miR-155 group compared with the WT group $(\mathrm{P}=0.013)$; however, this effect was abrogated in the AMO-155+miR-155 group $(\mathrm{P}=0.373)$. These data suggest that miR-155 functions upstream and inhibits the expression of SOCS-1 in astrocytes. As astrocytes are important effector cells in $\mathrm{ICH}$, the results of the in vitro experiments suggest that miR-155 contributes to ICH-induced inflammatory pathogenesis by inhibiting SOCS-1 expression.

\section{Discussion}

Based on the results of the present study, miR-155 may be required for the development of $\mathrm{ICH}$-induced inflammation in mice. Furthermore, the results of the present study revealed that miR-155 downregulates the expression levels of SOCS-1 protein, but increases the mRNA expression levels of the pro-inflammatory cytokines IFN- $\beta$, IL-6 and TNF- $\alpha$ in vivo. In addition, the association between miR-155 and SOCS-1 was demonstrated by transfection of astrocytes in vitro. This signaling pathway may be responsible for the effect of GCs on ICH-induced inflammation, as miR-155 expression was significantly suppressed, while SOCS-1 protein expression levels were significantly increased, when dexamethasone was administered to ICH mice. Previous studies have revealed that miR-155 is involved in biological processes, including inflammatory pathogenesis $(6,21,22)$; however, the role of miR-155 in ICH-induced inflammation and its specific signaling pathway remains to be elucidated. Although GCs have been demonstrated to be effective in controlling various inflammatory disorders $(9,36,37)$, the exact underlying mechanism of their anti-inflammatory effects remains unclear. The results of the present study provide, to the best of our knowledge, the first evidence of the involvement of miR-155 in ICH-induced inflammatory pathogenesis and the signaling pathway of GCs in this process. These results thus contribute to our understanding of the underlying mechanisms involved in ICH-induced inflammation.

The production of pro-inflammatory cytokines, including IFN- $\beta$, IL- 6 and TNF- $\alpha$, is an important downstream effect of ICH (1). Enhanced expression levels of IFN- $\beta$, IL- 6 and TNF- $\alpha$ mRNAs, accompanied by increased expression of miR-155 and reduced expression of SOCS-1 protein levels, indicates a critical role for the miR-155/SOCS-1 signaling cascade in ICH-induced inflammation. Previous studies have attributed the increased production of pro-inflammatory cytokines following ICH to the activation of Toll-like receptors (TLRs), particularly TLR2 and TLR4 (38). The classical signaling pathway triggered by TLRs results in the activation of nuclear factor (NF) $\kappa \mathrm{B}$ and mitogen-activated protein kinases (JNK and $\mathrm{p38}$ ), which leads to the transcription of pro-inflammatory cytokine genes (39-41). As miR-155 is transcribed from the BIC gene, the activation of which is under the control of
NF- $\kappa$ B, miR-155/SOCS-1 may function downstream of TLR signaling following ICH. This has previously been demonstrated in macrophage-mediated innate immune reactions (42). Nevertheless, additional studies are required to clarify the association between TLR signaling and the miR-155/SOCS-1 signaling cascade following ICH.

The significant decrease in the mRNA expression levels of IFN- $\beta$, IL- 6 and TNF- $\alpha$, combined with the decrease in miR-155 expression and enhanced expression levels of SOCS-1 protein following dexamethasone administration, suggests that GCs, including dexamethasone, function through the miR-155/SOCS-1 signaling pathway to attenuate ICH-induced inflammation. This underlying functional mechanism of dexamethasone is consistent with that of 1,25-dihydroxyvitamin $\mathrm{D}$, which inhibits TLR-mediated inflammation by targeting the miR-155/SOCS-1 signaling pathway in macrophages (42). Whether other regulatory mechanisms underlie GC function in $\mathrm{ICH}$ requires further investigation.

The expression levels of miR-155 and the pro-inflammatory cytokines in the ICH+DEX group, although inhibited compared with the ICH group, remained significantly increased compared with the control group. This suggests that GCs may produce adverse effects on brain pathology. Potential adverse effects caused by GCs have been reported previously, for instance on the hypothalamus-pituitary-adrenal axis $(43,44)$. Due to the beneficial effects of GCs, future studies are required to investigate their underlying functional mechanisms.

In conclusion, the results of the present study demonstrate an essential role of the miR-155/SOCS-1 signaling cascade in $\mathrm{ICH}$-induced inflammation, and furthermore reveal that GCs may relieve this inflammation by targeting the miR-155/SOCS-1 signaling pathway. Targeting the miR-155/SOCS-1 signaling pathway may provide a novel therapeutic strategy for the treatment of $\mathrm{ICH}$-triggered inflammation.

\section{Acknowledgements}

The present study was supported by the National Natural Science Foundation of China (grant nos. 81302617, 81172897, 81571848 and 81172898) and the Priority Academic Program Development of Jiangsu Higher Education Institutions.

\section{References}

1. Magistris FB, Bazak SB and Martin J: Intracerebral hemorrhage: Pathophysiology, diagnosis and management. Clinical Review 10: 14-22, 2013.

2. Derex L and Nighoghossian N: Intracerebral haemorrhage after thrombolysis for acute ischaemic stroke: An update. J Neurol Neurosurg Psychiatry 79: 1093-1099, 2008.

3. Wang C, Ji B, Cheng B, Chen J and Bai B: Neuroprotection of microRNA in neurological disorders (Review). Biomed Rep 2: 611-619, 2014.

4. Jickling GC, Ander BP, Zhan X, Noblett D, Stamova B and Liu D: microRNA expression in peripheral blood cells following acute ischemic stroke and their predicted gene targets. PLoS One 9: e99283, 2014.

5. Tan JR, Koo YX, Kaur P, Liu F, Armugam A, Wong PT and Jeyaseelan K: microRNAs in stroke pathogenesis. Curr Mol Med 11: 76-92, 2011.

6. Cardoso AL, Guedes JR, Pereira de Almeida L and Pedroso de Lima MC: miR-155 modulates microglia-mediated immune response by down-regulating SOCS-1 and promoting cytokine and nitric oxide production. Immunology 135: 73-88, 2012. 
7. Lansberg MG, Albers GW and Wijman CA: Symptomatic intracerebral hemorrhage following thrombolytic therapy for acute ischemic stroke: A review of the risk factors. Cerebrovasc Dis 24: 1-10, 2007.

8. Coutinho AE and Chapman KE: The anti-inflammatory and immunosuppressive effects of glucocorticoids, recent developments and mechanistic insights. Mol Cell Endocrinol 335 2-13, 2011.

9. Zheng Y, Xiong S, Jiang P, Liu R, Liu X, Qian J, Zheng X and Chu Y: Glucocorticoids inhibit lipopolysaccharide-mediated inflammatory response by downregulating microRNA-155: A novel anti-inflammation mechanism. Free Radic Biol Med 52: 1307-1317, 2012.

10. Spies CM, Strehl C, van der Goes MC, Bijlsma JW and Buttgereit F: Glucocorticoids. Best Pract Res Clin Rheumatol 25: 891-900, 2011

11. McMaster A and Ray DW: Modelling the glucocorticoid receptor and producing therapeutic agents with anti-inflammatory effects but reduced side-effects. Exp Physiol 92: 299-309, 2007.

12. Kreitzer N and Adeoye O: An update on surgical and medical management strategies for intracerebral hemorrhage. Semin Neurol 33: 462-467, 2013

13. Gu YT, Zhang H and Xue YX: Dexamethasone treatment modulates aquaporin- 4 expression after intracerebral hemorrhage in rats. Neurosci Lett 413: 126-131, 2007.

14. Vandevyver S, Dejager L, Tuckermann J and Libert C: New insights into the anti-inflammatory mechanisms of glucocorticoids: An emerging role for glucocorticoid-receptor-mediated transactivation. Endocrinology 154: 993-1007, 2013.

15. Jing J, Wu J, Liu W, Xiong S, Ma W, Zhang J, Wang W, Gui JF and Mei J: Sex-biased miRNAs in gonad and their potential roles for testis development in yellow catfish. PLoS One 9: e107946, 2014.

16. Lin F, Yao L, Xiao J, Liu D and Ni Z: MiR-206 functions as a tumor suppressor and directly targets K-Ras in human oral squamous cell carcinoma. Onco Targets Ther 7: 1583-1591, 2014.

17. Liu H, Yang Y, Zhang L, Liang R, Ge RS, Zhang Y, Zhang Q, Xiang Q, Huang Y and Su Z: Basic fibroblast growth factor promotes stem Leydig cell development and inhibits LH-stimulated androgen production by regulating microRNA expression. J Steroid Biochem Mol Biol 144: 483-491, 2014.

18. Ramirez-Salazar EG, Salinas-Silva LC, Vázquez-Manríquez ME, Gayosso-Gómez LV, Negrete-Garcia MC, Ramírez-Rodriguez SL, Chávez R, Zenteno E, Santillán P, Kelly-García J and Ortiz-Quintero B: Analysis of microRNA expression signatures in malignant pleural mesothelioma, pleural inflammation and atypical mesothelial hyperplasia reveals common predictive tumorigenesis-related targets. Exp Mol Pathol 97: 375-385, 2014.

19. Lu C, Huang X, Zhang X, Roensch K, Cao Q, Nakayama KI, Blazar BR, Zeng Y and Zhou X: miR-221 and miR-155 regulate human dendritic cell development, apoptosis, and IL-12 production through targeting of p27kip1, KPC1, and SOCS-1. Blood 117: 4293-4303, 2011

20. Jiang S, Zhang HW, Lu MH, He XH, Li Y, Gu H, Liu MF and Wang ED: MicroRNA-155 functions as an OncomiR in breast cancer by targeting the suppressor of cytokine signaling 1 gene. Cancer Res 70: 3119-3127, 2010.

21. Yao R, Ma YL, Liang W, Li HH, Ma ZJ, Yu X and Liao YH: MicroRNA-155 modulates Treg and Th17 cells differentiation and Th17 cell function by targeting SOCS1. PLoS One 7: e46082, 2012.

22. Kanwal N, John P and Bhatti A: MicroRNA-155 as a therapeutic target for inflammatory diseases. Rheumatol Int 33: 557-560, 2013

23. Liu DZ, Tian Y, Ander BP, Xu H, Stamova BS, Zhan X, Turner RJ, Jickling $G$ and Sharp FR: Brain and blood microRNA expression profiling of ischemic stroke, intracerebral hemorrhage, and kainate seizures. J Cereb Blood Flow Metab 30: 92-101, 2010.

24. Zhu GF, Yang LX, Guo RW, Liu H, Shi YK, Wang H, Ye JS, Yang ZH and Liang X: miR-155 inhibits oxidized low-density lipoprotein-induced apoptosis of RAW264.7 cells. Mol Cell Biochem 382: 253-261, 2013.

25. Xue H,Hua LM, Guo M and Luo JM: SHIP1 is targeted by miR-155 in acute myeloid leukemia. Oncol Rep 32: 2253-2259, 2014.
26. O'Connell RM, Chaudhuri AA, Rao DS and Baltimore D: Inositol phosphatase SHIP1 is a primary target of miR-155. Proc Natl Acad Sci USA 106: 7113-7118, 2009.

27. Chang P, Dong W, Zhang M, Wang Z, Wang Y, Wang T, Gao Y, Meng H, Luo B, Luo C, et al: Anti-necroptosis chemical necrostatin-1 can also suppress apoptotic and autophagic pathway to exert neuroprotective effect in mice intracerebral hemorrhage model. J Mol Neurosci 52: 242-249, 2014.

28. Wang T, Huang Y, Zhang M, Wang L, Wang Y, Zhang L, Dong W, Chang P, Wang Z, Chen X and Tao L: [Gly14]-Humanin offers neuroprotection through glycogen synthase kinase- $3 \beta$ inhibition in a mouse model of intracerebral hemorrhage. Behav Brain Res 247: 132-139, 2013.

29. McGlone JJ and Swanson J: Update on the guide for the care and use of agricultural animals in research and teaching. J Dairy Sci 93: 12,2010

30. Wang T, Zhang L, Zhang M, Bao H, Liu W, Wang Y, Wang L, Dai D, Chang P, Dong W, et al: [Gly14]-Humanin reduces histopathology and improves functional outcome after traumatic brain injury in mice. Neuroscience 231: 70-81, 2013

31. Luo X, Xiao J, Lin H, Li B, Lu Y, Yang B and Wang Z: Transcriptional activation by stimulating protein 1 and post-transcriptional repression by muscle-specific microRNAs of IKs-encoding genes and potential implications in regional heterogeneity of their expressions. J Cell Physiol 212: 358-367, 2007.

32. Roth-Cross JK, Martínez-Sobrido L, Scott EP, García-Sastre A and Weiss SR: Inhibition of the alpha/beta interferon response by mouse hepatitis virus at multiple levels. J Virol 81: 7189-7199, 2007.

33. Fang X, Hu H, Xie J, Zhu H, Zhang D, Mo W, Zhang R and Yu M: An involvement of neurokinin-1 receptor in FcERI-mediated RBL-2H3 mast cell activation. Inflamm Res 61: 1257-1263, 2012.

34. Tallant EA and Higson JT: Angiotensin II activates distinct signal transduction pathways in astrocytes isolated from neonatal rat brain. Glia 19: 333-342, 1997

35. Caplen NJ, Parrish S, Imani F, Fire A and Morgan RA: Specific inhibition of gene expression by small double-stranded RNAs in invertebrate and vertebrate systems. Proc Natl Acad Sci USA 98: 9742-9747, 2001.

36. van der Velden VH: Glucocorticoids: Mechanisms of action and anti-inflammatory potential in asthma. Mediators Inflamm 7: 229-237, 1998.

37. Schleimer RP: An overview of glucocorticoid anti-inflammatory actions. Eur J Clin Pharmacol 45 (Suppl 1): S3-S7; discussion S43-S44, 1993.

38. Wang YC, Zhou Y, Fang H, Lin S, Wang PF, Xiong RP, Chen J, Xiong XY, Lv FL, Liang QL and Yang QW: Toll-like receptor $2 / 4$ heterodimer mediates inflammatory injury in intracerebral hemorrhage. Ann Neurol 75: 876-889, 2014.

39. Arroyo DS, Soria JA, Gaviglio EA, Rodriguez-Galan MC and Iribarren P: Toll-like receptors are key players in neurodegeneration. Int Immunopharmacol 11: 1415-1421, 2011.

40. Kong Y and Le Y: Toll-like receptors in inflammation of the central nervous system. Int Immunopharmacol 11: 1407-1414, 2011.

41. Eberle ME and Dalpke AH: Dectin-1 stimulation induces suppressor of cytokine signaling 1, thereby modulating TLR signaling and $\mathrm{T}$ cell responses. J Immunol 188: 5644-5654, 2012.

42. Chen Y, Liu W, Sun T, Huang Y, Wang Y, Deb DK, Yoon D, Kong J, Thadhani R and Li YC: 1,25-Dihydroxyvitamin D promotes negative feedback regulation of TLR signaling via targeting microRNA-155-SOCS1 in macrophages. J Immunol 190: 3687-3695, 2013.

43. Li ZQ, Liang GB, Xue YX and Liu YH: Effects of combination treatment of dexamethasone and melatonin on brain injury in intracerebral hemorrhage model in rats. Brain Res 1264: 98-103, 2009

44. Lema PP, Girard C and Vachon P: Evaluation of dexamethasone for the treatment of intracerebral hemorrhage using a collagenase-induced intracerebral hematoma model in rats. J Vet Pharmacol Ther 27: 321-328, 2004. 